\section{Le modèle \\ managérial \\ de la recherche}

Critique et alternative

Romain Brette
$>$ Faire de la science est un engagement moral: s'efforcer de dire le vrai sur le monde. Le modèle managérial de la recherche, fondement idéologique des réformes du système de recherche engagées en France depuis les années 2000, s’y oppose frontalement. II consiste à identifier le chercheur à un homo economicus cherchant en premier lieu à maximiser son intérêt personnel, qu'une bureaucratie tente d'aligner sur des objectifs de performance chiffrés. Ce modèle est incohérent et destructeur. La science est permise par la curiosité, l'émulation et l'éthique intellectuelle. Ce sont donc ces traits humains qu'une organisation rationnelle de la recherche doit tenter de favoriser et d'exploiter. <

\section{Une perspective historique}

L'organisation de la recherche scientifique, et en particulier biomédicale, a profondément changé en quelques décennies. David Hubel, prix Nobel de physiologie ou médecine pour ses découvertes sur le système visuel, avec son collègue Thorsten Wiesel, décrit ainsi ce changement: "Aujourd'hui, la recherche biomédicale est portée par des groupes constitués d'un directeur entouré de 5-20 étudiants et postdocs. Cela contraste avec la situation passée, où la recherche était habituellement faite par des individus ou de petits groupes de deux ou trois scientifiques qui concevaient et implémentaient la recherche eux-mêmes. Aujourd'hui le chercheur titulaire passe son temps dans un bureau à faire de l'administration [...] » [1].

Ainsi, dans la revue Nature, le nombre médian d'auteurs d'un article est passé de 1, en 1945, à 16, en 2008, avec une accélération dans les années 2000 [2]. Cette évolution s'est produite dans toutes les disciplines, à des degrés divers, et elle coïncide avec une diminution notable de l'autonomie des jeunes chercheurs. Aux États-Unis, l'âge d'obtention du premier financement

Vignette (ㄷ Thierry Jouault)

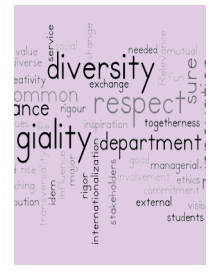

\author{
Sorbonne Université, \\ UPMC Univ Paris 6, Inserm, \\ CNRS, Institut de la vision, \\ 17 rue Moreau, 75012 Paris, \\ France. \\ romain.brette@inserm.fr
}

(R01) est passé de 35 ans en 1970 à 42 ans au début des années 2000 [3]. Cette évolution est également manifeste en France dans les recrutements de chercheurs et d'enseignants-chercheurs, où l'âge de recrutement comme chargé de recherche ou comme maître de conférences a augmenté de 2 ans ces dix dernières années [4]. Or, si le métier de chercheur a toujours nécessité une longue phase d'apprentissage [5], celle-ci n'impliquait pas nécessairement, surtout pour les post-doctorants, de travailler sous les ordres d'un directeur.

Lorsqu'en 1939, Alan Hodgkin et Andrew Huxley (futurs prix Nobel) publient dans Nature la première mesure intracellulaire du potentiel d'action dans un nerf [6], ils ont respectivement 25 et 22 ans. Dans leur écrit, ils remercient simplement le zoologiste John Young, pour avoir «rendu ce travail possible». Dix ans plus tard, Alan Hodgkin invite Bernard Katz, un biophysicien de sa génération (et autre futur prix Nobel), pour une collaboration expérimentale dans le laboratoire de biologie marine de Plymouth où il travaille. Dans leur publication commune, dont ils sont seuls auteurs, ils remercient le directeur (sans même le nommer) et le personnel du laboratoire «pour leur hospitalité et les généreux équipements mis à leur disposition » [7]. Une telle autonomie semble aujourd'hui presque incongrue; elle a pourtant permis une bonne partie des avancées majeures en biologie.

Cette situation a changé non par l'évolution naturelle de la science, mais sous l'impulsion de deux grandes transformations structurelles d'origine politique. La première est le financement par projet. Aux États-Unis, l'Agence de financement nationale (National Science 
Foundation, NSF) est née en 1950 dans un contexte de guerre froide. Il s'agissait d'une part de financer la guerre froide, mais également, sur le plan idéologique, de concevoir un modèle de financement le plus éloigné possible de la planification soviétique [8]. Le financement ne prend alors plus la forme de dotations, mais de contrats, avec des engagements. Cependant, l'année de sa création, la NSF ne finançait que 24 projets. De même, si les NIH (National Institutes of Health) existaient depuis plus longtemps, le financement de projets ne représentait pas alors une activité importante. Au cours de la guerre froide, ce mode de financement a pris une ampleur considérable, la NSF finançant plus de 10000 projets à partir de 1983, les NIH environ 50000 projets en 2020. En France, le financement par projet a pris un rôle majeur lors de la création en 2005 de l'Agence nationale de la recherche (ANR). Ces financements permettent en particulier de recruter de jeunes chercheurs, notamment des post-doctorants, qui devront implémenter le projet. Or il ne s'agit plus ici pour le jeune chercheur d'obtenir une bourse pour poursuivre ses recherches, comme il en existe depuis longtemps, mais d'effectuer une tâche d'un projet conçu par un chercheur établi, selon un engagement contractuel. L'étudiant et le jeune chercheur deviennent des ressources humaines.

La deuxième évolution majeure est la transformation managériale des universités [9]. Elle repose en particulier sur la théorie du nouveau management public, d'abord mise en place par la Première ministre Margaret Thatcher au Royaume-Uni, puis généralisée aux autres pays occidentaux. Cette théorie entend calquer le fonctionnement d'un service public sur celui d'une entreprise commerciale, présumée « efficace » grâce aux mécanismes du marché. II s'agit en particulier de gérer les services publics par le biais d'objectifs chiffrés. Or un service public ne disposant pas naturellement de cette mesure objective d'efficacité qu'est le profit, des mesures doivent être créées de toutes pièces - nombre d'articles publiés, h-index, classements internationaux, nombre de brevets, etc. La théorie du nouveau management public repose donc sur une faille conceptuelle majeure: le sophisme dualiste consistant à déplacer le sujet à expliquer hors du champ d'explication. Comment produire de la bonne science ? En optimisant une mesure de la qualité de la science. Mais qu'est-ce que la «qualité de la science »? Peut-on la mesurer, et si oui, comment? Ces questions fondamentales sont présumées résolues d'emblée, autrement dit magiquement, par cette âme du système de recherche qu'est la bureaucratie. Cette faille conceptuelle explique pourquoi cette idéologie d'inspiration libérale aboutit paradoxalement à un accroissement de la bureaucratie [10].

Ces transformations d'origine idéologique aboutissent au modèle managérial aujourd'hui dominant, au moins dans le monde anglosaxon, où un individu, que l'on nomme PI (pour principal investigator, en français, investigateur principal), gère son laboratoire comme une petite entreprise, en compétition avec d'autres laboratoires-entreprises. L'activité du laboratoire est considérée comme une production qui peut être quantifiée. Pour financer cette production, le PI obtient des financements de projets, dans lesquels on explicite les ressources pour accomplir des tâches, et en particulier des ressources humaines: des étudiants et des post-doctorants, et quelques techniciens. Ce modèle contraste avec le modèle historique français dans lequel le chercheur et l'enseignant-chercheur sont recrutés de manière précoce, peu de temps après leur soutenance de thèse (et depuis 1983 sur un statut de fonctionnaire ${ }^{1}$ ), et où la recherche était financée principalement sur dotation. Ce modèle historique s'est progressivement transformé depuis le début des années 2000 , avec la contractualisation d'un nombre croissant de personnels (les jeunes chercheurs en particulier), le remplacement progressif du financement récurrent par des appels à projet, le développement de l'évaluation managériale couplée à des systèmes de primes (de recherche ou d'encadrement).

Comme évoqué précédemment, l'hégémonie du modèle managérial de la recherche ne repose pas sur une supériorité démontrée, dont la base empirique est en effet inexistante [11], mais sur des présupposés idéologiques dont on peut aisément exposer l'incohérence.

\section{Le chercheur, une ressource humaine?}

Commençons par une remarque élémentaire. Dans un système stable, un chercheur forme en moyenne exactement un nouveau chercheur dans la totalité de sa carrière. Prenons un exemple concret. Supposons que l'équipe typique d'un PI soit constituée de 3 doctorants, soit nettement moins que ce que rapporte David Hubel. Si un doctorat dure quatre ans et que la carrière d'un chercheur en dure 40, alors le PI aura formé 30 doctorants au cours de sa carrière, dont un seul lui succèdera. Un doctorant a donc une chance sur 30 d'obtenir un poste stable. Si l'on y ajoute deux post-doctorants et que le post-doctorat dure en moyenne quatre ans, alors chaque post-doctorant a une chance sur 20 de devenir PI. On voit donc bien que le problème de la précarité des jeunes chercheurs ne peut être résolu en faisant en sorte que davantage de jeunes chercheurs puissent devenir $\mathrm{Pl}$ et ouvrir leur propre laboratoire. C'est le modèle managérial lui-même qui, indépendamment des considérations budgétaires, produit cette situation. De fait, le budget des NIH a doublé au début des années 2000, après quoi le taux de succès aux financements de projet a été divisé par deux et l'âge du premier financement a augmenté [12].

Cette précarité ne serait-elle pas «un mal pour un bien », par les effets vertueux d'une compétition accrue? II s'agit ici d'une logique d'excellence, c'està-dire de concentration des ressources sur les meil-

1 Décret n83-1260 du 30 décembre 1983, pour les chercheurs des EPST (Établissements publics à caractère scientifique et technologique) et décret $n^{\circ} 84-431$ du 6 juin 1984, pour les enseignants-chercheurs. 
leurs. Or cette logique est tout à fait paradoxale lorsque les ressources sont principalement des ressources humaines. En effet, en confiant les ressources aux excellents, ceux-ci vont recruter des personnes qui, par construction, seront moins qualifiées, et surtout moins expérimentées. Concentrer les ressources sur les excellents a donc pour conséquence directe de diminuer la qualification globale du personnel de recherche. Par ailleurs, plusieurs études empiriques montrent qu'il est plus efficace de diversifier les financements que de les concentrer en créant de grosses structures [13-16].

On pourrait objecter qu'un doctorant est un étudiant, qui n'a pas forcément vocation à devenir chercheur. On pourrait par exemple considérer que le doctorat est une formation à l'autonomie scientifique, qui pourrait être utile pour travailler dans l'industrie, ou pour faire face dans l'administration à des défis de santé publique, comme par exemple des épidémies mortelles. L'objection est juste, et montre précisément le danger de considérer l'étudiant comme une ressource humaine, c'est-à-dire quelque chose qui est utilisé en vue d'un but productif, en l'occurrence implémenter une tâche d'un projet de recherche. Le modèle managérial réduit donc la formation au strict nécessaire pour l'implémentation du projet, et évacue toute autre finalité éducative du doctorat, telle que le développement de l'esprit critique ou de l'autonomie scientifique. Bien sûr, le chercheur consciencieux aura sans doute à cœur de dispenser néanmoins cette formation. Mais l'on voit qu'il s'agit là d'un acte éthique, directement découragé par la structure puisque le chercheur est mis en compétition avec d'autres chercheurs. Autrement dit, un acte de résistance.

\section{Modèle marchand et éthique intellectuelle}

Faire de la science, c'est rechercher la vérité sur le monde ; or la vérité est inconnue avant d'avoir été établie. De ces deux platitudes découle l'inadéquation radicale du modèle marchand pour décrire l'activité scientifique.

Dans le modèle marchand, le bon fonctionnement du système repose non pas sur l'éthique des agents, mais sur leur intérêt personnel, en particulier financier. Pour que cela soit possible, il faut que, dans une transaction marchande, l'acheteur dispose d'une information complète et objective sur le produit [17]. Ce modèle ne peut donc pas s'appliquer à l'information elle-même, puisque l'acheteur ne peut pas à la fois disposer au préalable de cette information et chercher à l'acquérir. On ne peut donc pas comparer la production de connaissances à une production marchande.

La science a une autonomie épistémique, c'est-à-dire qu'il n'existe pas de normes extérieures à la science permettant d'évaluer la véracité des propositions scientifiques. Ceci est du ressort du débat scientifique, donc d'un processus interne à la science. II s'ensuit que «faire de la science » est en premier lieu un engagement d'ordre éthique, celui de tenter de dire le vrai [18], et qui doit idéalement prendre le pas sur toute considération d'ordre personnel. II ne s'agit pas ici de dire que la science énonce des vérités. Chacun sait qu'une des caractéristiques du discours scientifique est qu'il est révisable, donc potentiellement faux. Mais si on le révise, c'est justement que l'on considère la vérité comme une valeur normative qui doit guider l'activité scientifique. Sans cette valeur partagée, il ne peut y avoir ni progrès scientifique, ni même de débat scientifique possible - débattre suppose que l'on soit attaché à une notion de vérité qui dépasse sa propre personne. Si la science n'est pas identifiable à la vérité, on peut donc résumer que l'activité scientifique est la poursuite de la vérité.

Naturellement, cette remarque ne s'applique pas qu'à la science. La médecine, par exemple, reconnaît explicitement le fondement éthique du métier par le serment d'Hippocrate, c'est-à-dire qu'il s'agit de soigner et non simplement de maximiser des flux de patients.

Organiser la recherche, c'est donc en premier lieu concevoir des structures propices au déploiement de l'éthique intellectuelle. Cette ambition s'oppose frontalement au point de vue managérial, qui consiste à concevoir des structures d'incitation, c'est-à-dire un ensemble d'incitations financières et de sanctions couplées à des métriques à optimiser.

\section{Un autre laboratoire est possible}

Une organisation sociale présuppose un certain modèle de l'être humain. Le modèle managérial considère que l'homme est mu exclusivement par la peur et l'avidité - le fameux homo economicus qui, rappelons-le, est une espèce fictionnelle. Or ce modèle n'est pas seulement faux; en effet, il existe d'autres passions humaines - le psychologue Mihály Csíkszentmihályi, par exemple, a passé une partie de sa vie à essayer de comprendre pourquoi les gens font volontairement du ski [19]. "Tous les modèles sont faux, mais certains sont utiles », écrivait George Box [20]. Le modèle de l'homo economicus est également inutile, puisqu'un modèle qui ne laisse aucune place à l'éthique intellectuelle ne peut pas rendre compte de l'activité intellectuelle.

On devine dans les exemples historiques discutés précédemment que d'autres passions humaines étaient à l'œuvre: la curiosité, la camaraderie, l'excitation de la découverte, peut-être l'orgueil, et bien sûr, le sens moral qui pousse à rapporter honnêtement et rigoureusement ce que l'on croit être vrai.

Joseph Rogers Hollingsworth (Université du Wisconsin) a tenté de comprendre ce qui distingue, principalement aux États-Unis, les lieux où se sont faites des découvertes majeures [21]. II s'agit en résumé de structures qui favorisent les interactions sociales entre scientifiques. De taille relativement modeste, peu bureaucratiques, peu hiérarchiques, ces endroits ont une diversité scientifique importante mais pas illimitée, de sorte 
que les scientifiques puissent effectivement communiquer entre eux. L'intégration sociale est favorisée par des repas pris en commun, des clubs de lecture d'articles. Il ne s'agit pas ici de primes et de sanctions, ni d'appels d'offre compétitifs; il s'agit de conditions structurelles favorisant certains comportements sociaux [29] $(\rightarrow)$.

On sait qu'un autre laboratoire est possible, $(\rightarrow)$ Voir le Forum de J. Haiech et T. Jouault, $m / s$ $n^{\circ}$ 6-7, juin-juillet 2020, page 651 puisque le modèle managérial est relativement récent. II ne s'agit pas pour autant d'idéaliser les systèmes passés. Si la création en France de l'ANR a été soutenue par une partie de la communauté scientifique, c'est qu'elle était perçue comme une alternative à un modèle dirigiste, hiérarchique, dans lequel la Direction décide de l'allocation des ressources selon des critères qui lui sont propres, empêchant l'autonomie des jeunes chercheurs et l'émergence d'idées originales. Le modèle du financement par projet a ainsi un attrait non négligeable: c'est un modèle décentralisé avec une apparence de collégialité, puisque les projets sont évalués par les pairs. Cependant, en concentrant les ressources sur des chercheurs devenus employeurs, on reconstruit, de fait, un modèle hiérarchique.

Les défauts du financement par projet sont bien documentés. II ne suffit pas que l'évaluation soit faite par les pairs: encore faut-il que la base de cette évaluation ait un sens. En l'occurrence, cette base est la description de toutes les étapes intermédiaires nécessaires à la découverte d'un savoir préalablement inconnu; cela peut sembler audacieux. On sait par exemple que ce système défavorise la recherche interdisciplinaire [22]. Fondamentalement, un système de sélection par comité freine nécessairement l'originalité pour une raison très simple : un projet sélectionné par consensus est un projet consensuel. Demander à un comité de choisir des projets non consensuels est une injonction paradoxale. On sait également qu'en plus des biais, la sélection par projets a une très forte composante aléatoire [23-25], et que lorsque le taux de sélection est faible, le coût du système (écriture et évaluation des projets) est supérieur aux montants financés [26]. Ces défauts avérés ont conduit certains scientifiques à proposer de remplacer ce système par une «loterie », de façon à réduire les biais ainsi que le travail inutile du comité [27]. Bien que cette proposition soit, semble-t-il, tout à fait sérieuse, et a d'ailleurs été implémentée en Nouvelle-Zélande [28], elle n'en constitue pas moins une véritable réduction à l'absurde. La justification initiale de l'inégalité dans l'accès aux ressources est qu'elle est nécessaire à une allocation optimale des ressources. Si cette allocation devient aléatoire, la justification devient caduque.

Il est tout à fait possible d'imaginer un système alternatif qui ne soit ni dirigiste, ni managérial. Pour le montrer, je vais examiner une proposition simple qui n'a pas l'ambition d'être exhaustive ni bien délimitée, mais qui a simplement celle d'ouvrir un champ de possibilités. Chaque chercheur reçoit d'emblée un budget - il y a en effet une certaine logique à donner à chaque personne recrutée les moyens minimaux pour travailler. On finance par ailleurs des infrastructures mutualisées: par exemple, un service public de publication académique, des plateformes expérimentales, une machine à café. Les chercheurs discutent entre eux, et de ces discussions naissent des envies de collaboration. Pour travailler ensemble, les chercheurs mettent en commun leurs ressources. Cette mise en commun des ressources remplace la procédure bureaucratique de financement d'un projet.

Examinons maintenant les vertus et difficultés de cette proposition. Premièrement, il s'agit d'un système qui n'est pas dirigiste. Au contraire, il préserve l'autonomie scientifique, et donc favorise l'originalité. Deuxièmement, ce système conserve le principe de sélection par les pairs, mais avec une différence importante: celui qui accepte le «projet » est, d'une part, expert du sujet, puisqu'il est capable d'y participer, et, d'autre part, a d'autant plus intérêt à en faire une évaluation correcte qu'il va faire une partie du travail et apporter ses propres ressources. Troisièmement, cette proposition fait l'économie de la bureaucratie, ce qui d'une part économise des ressources et du temps, permettant au chercheur d'exercer effectivement son métier de chercheur, et d'autre part réduit à néant le délai entre la conception d'un «projet » et son financement, ce qui peut permettre, par exemple, de réagir rapidement à une pandémie mondiale. Quatrièmement, au lieu de recruter du personnel temporaire, on sollicite des pairs. La qualification du personnel est donc augmentée.

Naturellement, cette proposition n'est pas sans difficultés, mais le point crucial est que ces difficultés ne sont pas mieux résolues par le système d'appels à projet. Ainsi, on peut objecter que, dans ce système, les ressources sont proportionnelles au nombre de personnes participant au projet, au lieu d'être adaptées aux besoins de ce projet. Cette objection est pertinente, mais s'applique tout autant au système de financement par projets. Chaque appel à projets a un budget attendu, dans une fourchette assez restreinte, car c'est une condition nécessaire pour pouvoir comparer les mérites des différentes candidatures. Ce sont donc les projets qui s'adaptent aux ressources, et non le contraire. Pire, le financement compétitif incite le chercheur à faire des demandes multiples, de sorte que les ressources allouées sont alternativement insuffisantes ou dispendieuses. C'est donc un système par construction très médiocre en termes d'allocation des ressources. Quant au système de mise en commun des ressources, on peut tout à fait imaginer le compléter par un processus de demande de fonds exceptionnels avec évaluation formelle, sans qu'il constitue le cœur du système.

Dans le même ordre d'idée, on peut également objecter que les besoins peuvent fluctuer d'une année à l'autre. Par exemple, un chercheur pourrait décider d'occuper son année à écrire un livre, auquel cas, il n'aurait pas besoin de son budget. Mais là encore, le système du 
financement par projets ne règle pas ce problème, d'une part parce qu'il a une grande inertie (la durée des financements contractuels), et d'autre part parce qu'il incite à soumettre des demandes de financement continuellement pour s'assurer contre les fluctuations aléatoires du financement compétitif. Une conséquence habituelle est la liquidation en fin de projet du budget résiduel dans des dépenses modérément utiles.

Il existe une solution simple à ce problème, qui est la thésaurisation : on reporte le budget sur l'année suivante. Rien n'empêche d'imaginer des mécanismes de banque plus sophistiqués - par exemple, on épargne et emprunte par l'intermédiaire de parts dont la valeur est ajustée pour équilibrer les flux.

En résumé, le modèle managérial de la recherche est un modèle profondément dysfonctionnel, parce qu'il rentre en conflit direct avec la nature même de la science. Le rôle des structures sociales est de permettre à certains aspects de la motivation humaine de s'exprimer, plutôt que d'autres. Pour la science, ces motivations à encourager ne sont pas l'intérêt personnel, mais bien la curiosité, l'émulation intellectuelle et, surtout, l'éthique intellectuelle. $\diamond$

\section{SUMMARY}

\section{A critique of the managerial model of research}

To be a scientist is to make an implicit ethical commitment: to try to tell the truth about the world. The managerial model of research, which is the ideological foundation of modern political reforms of the research system around the world, stands in direct conflict with this assertion. It consists in identifying the scientist with a homo economicus looking to maximize its own profit, which a bureaucracy is tasked to align with performance objectives. This model is incoherent and destructive. Science is made possible by curiosity, emulation and intellectual ethics. These are the human traits that a rational research organization should try to favor and exploit. $\diamond$

\section{LIENS D'INTÉRÊT}

L'auteur déclare n'avoir aucun lien d'intérêt concernant les données publiées dans cet article.

\section{RÉFÉRENCES}

1. Hubel DH. The Way Biomedical Research Is Organized Has Dramatically Changed Over the Past Half-Century : Are the Changes for the Better? Neuron 2009; 64 : 161-3.

2. Monastersky R, van Noorden R. 150 years of Nature : a data graphic charts our evolution. Nature $2019 ; 575: 22-3$.

3. Gomez Diaz M. Unintended effects of changes in NIH appropriations : Challenges for biomedical research workforce development. PhD Thesis, Massachusetts Institute of Technology. 2012.

4. L'état de l'emploi scientifique en France-Édition 2020. Ministère de l'Enseignement supérieur, de la Recherche et de I'Innovation. www.enseignementsup-recherche.gouv.fr/cidl54848/Ietat-de-I-emploi-scientifique-en-france-edition-2020.html
5. Enders J, Musselin C. Back to the future? The academic professions in the 21 st century. High Educ To $2008 ; 2030: 125-50$.

6. Hodgkin A, Huxley A. Action Potentials Recorded from Inside a Nerve Fibre. Nature $1939 ; 144: 710$.

7. Hodgkin AL, Katz B. The effect of sodium ions on the electrical activity of the giant axon of the squid. J Physiol 1949; $108: 37$.

8. Kaiser D. The search for clean cash. Nature $2011 ; 472: 30-1$.

9. Ferlie $\varepsilon$, Musselin C, Andresani G. The steering of higher education systems : a public management perspective. High Educ $2008 ; 56: 325$.

10. Hibou B. La bureaucratisation du monde à l'ère néolibérale. Paris : La Découverte, 2012

11. Gläser J, Laudel G. Governing Science : How Science Policy Shapes Research Content. Eur J Sociol Arch Eur Sociol 2016; 57 : 117-68.

12. Couzin J, Miller G. Boom and Bust. Science 2007 ; 316 : 356-61.

13. Cook I, Grange $S$, Eyre-Walker A. Research groups: How big should they be? PeerJ 2015 ; 3. doi : 10.7717 /peerj. 989

14. Fortin JM, Currie DJ. Big Science vs. Little Science : How Scientific Impact Scales with Funding. PLoS ONE $2013 ; 8$.

15. Mongeon P, Brodeur C, Beaudry C, Lariviere V. Concentration of research funding leads to decreasing marginal returns. Res Eval 2016 ; rvw007.

16. Wahls WP. The NIH must reduce disparities in funding to maximize its return on investments from taxpayers. elife; 7 .

17. Rosser JB Jr. A Nobel Prize for Asymmetric Information : The economic contributions of George Akerlof, Michael Spence and Joseph Stiglitz. Rev Polit Econ $2003 ; 15: 3-21$.

18. Engel P. Les Vices du savoir : Essai d'éthique intellectuelle. Paris: Agone, 2019.

19. Csikszentmihalyi M. Creativity: Flow and the Psychology of Discovery and Invention. Ist edition. New York: Harper Perennial, 2013.

20. Box GEP. Robustness in the Strategy of Scientific Model Building. In : Launer RL, Wilkinson GN, editors. Robustness in Statistics. Academic Press, 1979. pp. 201-36.

21. Hollingsworth JR. Scientific Discoveries: An Institutionalist and PathDependent Perspective. 2008. In Caroline Hannaway, ed., Biomedicine in the Twentieth Century: Practices, Policies, and Politics. Bethesda, MD: National Institutes of Health, 2008. pp. 317-53.

22. Bromham L, Dinnage $R$, Hua $X$. Interdisciplinary research has consistently lower funding success. Nature $2016 ; 534: 684-7$.

23. Graves N, Barnett AG, Clarke P. Funding grant proposals for scientific research : retrospective analysis of scores by members of grant review panel. BMJ $2011 ; 343:$ d4797.

24. Fang FC, Bowen A, Casadevall A. NIH peer review percentile scores are poorly predictive of grant productivity. Elife 2016 ; 5 : el3323.

25. Scheiner SM, Bouchie LM. The predictive power of NSF reviewers and panels. Front Ecol Environ 2013 ; 11 : 406-7.

26. Gross K, Bergstrom CT. Contest models highlight inherent inefficiencies of scientific funding competitions. PLOS Biol $2019 ; 17$ : e3000065.

27. Fang FC, Casadevall A. Research Funding: the Case for a Modified Lottery. mBio $2016 ; 7$.

28. Liu M, Choy V, Clarke P, Barnett A, Blakely T, Pomeroy L. The acceptability of using a lottery to allocate research funding : a survey of applicants. Res Integr Peer Rev $2020 ; 5: 3$.

29. Haiech J, Jouault T. Think small! Science disruptive et petites équipes. Med Sci (Paris) $2020 ; 36: 651-3$.

\section{Retrouvez toutes les Actualités de la Myologie sur les sites de :}

la Société Française de Myologie

www.sfmyologie.org sfm

la filière de santé neuromusculaire FILNEMUS

www.filnemus.fr
TIRÉS À PART

R. Brette 